\title{
Long non-coding RNA NR_002794 is upregulated in pre-eclampsia and regulates the proliferation, apoptosis and invasion of trophoblast cells
}

\author{
YINYAO MA, XUXIA LIANG, HUA WU, CHUN ZHANG and YANHUA MA \\ Department of Obstetrics, People's Hospital of Guangxi Zhuang Autonomous Region, Nanning, Guangxi 530021, P.R. China
}

Received December 10, 2018; Accepted May 17, 2019

DOI: $10.3892 / \mathrm{mmr} .2019 .10701$

\begin{abstract}
Pre-eclampsia is a common complication during pregnancy, characterized by hypertension and proteinuria. The pathogenesis of pre-eclampsia is not fully understood. Studies on the maternal spiral artery have led scientists to consider that the ineffective infiltration of placental trophoblast cells may be a primary cause of pre-eclampsia. The present study aimed to investigate the differences in the profiles of long non-coding RNAs (lncRNAs) between the placentas of patients with pre-eclampsia and those of healthy pregnant women. The involvement of the differentially expressed lncRNAs in the biological activity of trophoblast cells was also assessed. A total of 26 differentially expressed lncRNAs were identified between the pre-eclampsia and healthy groups. Upregulation of NR_002794 was found in tissues from patients with pre-eclampsia. In SWAN71 trophoblast cells, NR_002794 had suppressive effects on proliferation and migration, and resulted in an increased rate of apoptosis. Furthermore, lncRNA NR_002794 had no effect on the phagocytosis of trophoblast cells. The present study suggested that abnormal levels of NR_002794 may lead to atypical conditions in trophoblast cells, which may be associated with the failure of maternal spiral artery remodeling during pregnancy and, consequently, with the development of pre-eclampsia.
\end{abstract}

\section{Introduction}

Pre-eclampsia is characterized by hypertension and proteinuria during pregnancy, and is prevalent in $3-5 \%$ of all pregnancies worldwide $(1,2)$. Pre-eclampsia affects maternal health, birth rate and neonatal mortality (3-5). Pregnant women with

Correspondence to: Dr Yinyao Ma or Dr Xuxia Liang, Department of Obstetrics, People's Hospital of Guangxi Zhuang Autonomous Region, 6 Taoyuan Road, Qingxu, Nanning, Guangxi 530021, P.R. China

E-mail: mayinyao66@126.com

E-mail: xuxialiang@yandex.com

Key words: long non-coding RNA NR_002794, trophoblast cells, pre-eclampsia, apoptosis, invasion pre-eclampsia are at risk of suffering a stroke, pulmonary edema and renal failure, and have shown symptoms of postpartum depression (6). They are also more susceptible to a variety of chronic diseases, including cardiovascular diseases later in life (6). Pre-eclampsia may also cause uterine fetal developmental limitations and premature birth. Infants born to mothers with pre-eclampsia have a higher incidence of cerebral palsy and bronchopulmonary dysplasia (5). Despite previous studies, the pathogenesis of pre-eclampsia remains elusive, with the most effective treatment being the immediate termination of pregnancy $(7,8)$.

Although the pathogenesis of pre-eclampsia is not fully understood, it is thought that abnormal placental development plays a significant role in the disease (9). During the normal remodeling of the maternal spiral artery, extravillous trophoblasts (EVTs) differentiate and invade the maternal endometrium, thus causing endovascular transformation $(10,11)$. Failure in the differentiation and invasion of trophoblastic cells may cause inadequate remodeling of the maternal spiral arteries, incomplete development of placental vessels and insufficient blood perfusion, an important cause of pre-eclampsia $(11,12)$. However, the detailed molecular mechanisms involved in this process have not been fully explored.

In the absence of the complete open reading frames necessary for protein synthesis, long non-coding RNAs (lncRNAs) are unable to encode proteins; however, they can carry out an array of biological functions associated with the regulation of gene expression (13). lncRNAs have been associated with various types of cancer, cardiovascular and neurodegenerative diseases, and are involved in the growth and development of several organisms $(14,15)$. Previous studies have shown that IncRNAs are involved in the invasion of trophoblast cells $(9,11)$. By determining the expression profile of lncRNAs in pre-eclamptic and normal placentas, He et al (16) suggested that IncRNAs may be involved in the pathogenesis of this condition. Previous studies have discussed the role of the abnormal expression of maternally expressed 3 (MEG3), metastasis associated lung adenocarcinoma transcript 1 (MALAT-1), Hox transcript antisense RNA and SPRY4 intronic transcript 1 (SPRY4-IT1) lncRNAs in the pathogenesis of pre-eclampsia $(11,12,17,18)$.

In the present study, the lncRNA and mRNA expression profiles were examined from tissues derived from the 
placentas of 3 patients with pre-eclampsia and 3 healthy patients. Thereafter, the function of NR_002794 was investigated as its expression was found to differ between the aforementioned samples with respect to trophoblast biology. The present study suggested that high levels of NR_002794 may be associated with the atypical conditions of trophoblast cells in pre-eclampsia; as such, NR_002794 may be a potential biomarker for the diagnosis of pre-eclampsia.

\section{Materials and methods}

Microarray assay and bioinformatics analysis. Total RNA was extracted using TRIzol ${ }^{\circledR}$ reagent (Thermo Fisher Scientific, Inc.), according to the manufacturer's instructions. To detect the expression profiles of lncRNAs and mRNAs in the placental tissues of patients with pre-eclampsia and in patients with normal pregnancies, microarray analysis was performed on lncRNAs and mRNAs in 3 pre-eclamptic and 3 matched control subjects (Affymetrix; Thermo Fisher Scientific, Inc.) (19). The placental tissues of the patients (median age, 29; range, 25-36) with pre-eclampsia and those with normal pregnancies were collected after parturition at the People's Hospital of Guangxi Zhuang Autonomous Region between April 2016 and September 2017. The population analyzed in the present study were pregnant women who had a gestational age of more than 20 weeks. Written consent was obtained from all donors. Gene ontology (GO) and Kyoto Encyclopedia of Genes and Genomes (KEGG) pathway analyses were used to investigate differentially expressed mRNAs and lncRNAs related to pre-eclampsia (20). DAVID version 6.8 (https://david.ncifcrf.gov/) was used to analyze large gene lists. These analyses were applied to investigate the functional enrichment and clustering of the differentially expressed mRNAs and lncRNAs.

Cell culture. SWAN71 first-trimester human trophoblasts and 293 T cells were cultured in DMEM medium supplemented with $10 \%$ FBS (both Gibco; Thermo Fisher Scientific, Inc.) at $37^{\circ} \mathrm{C}$ in a humidified atmosphere with $5 \% \mathrm{CO}_{2}$. SWAN71 cells (derived using telomerase-mediated transformation of a 7 weeks old cytotrophoblast isolate) were kindly provided by Professor Ke Wu (School of Life Sciences, Peking University, China) and 293T cells were purchased from the American Type Culture Collection.

Vector construction and lentiviral infection. In order to overexpress NR_002794, a pcDNA3.1 plasmid (Promega Corporation) was constructed expressing full-length NR_002794 (213 bp); the empty pcDNA3.1 plasmid was used as the control. The two plasmids (100 nM) were co-transfected into $293 \mathrm{~T}$ cells with a packaging plasmid (psPAX2; Promega Corporation) using Lipofectamine ${ }^{\circledR} 2000$ (Invitrogen; Thermo Fisher Scientific, Inc.). NR_002794 overexpression lentivirus (lenti-NR_002794) and a negative control lentivirus (lenti-control) were obtained after 3 days. Thereafter, the SWAN71 trophoblast cells were infected with $2 \times 10^{6}$ transducing units of lenti-NR_002794 and lenti-control. The cells were collected to determine infection efficiency at $48 \mathrm{~h}$ post-infection following incubation at $37^{\circ} \mathrm{C}$.
$R N A$ extraction and reverse transcription-quantitative PCR. Total RNA was extracted from placental tissues or cultured cells using RNA-ISOPLUS (Takara Bio, Inc.), according to the manufacturer's instructions. Complementary DNA (cDNA) was produced using a PrimeScript ${ }^{\mathrm{TM}}$ RT Reagent with gDNA Eraser (Perfect Real Time) kit (Takara Bio, Inc.) and qPCR was performed using SYBR Green (Takara Bio, Inc.). RT reactions were performed as follows: $42^{\circ} \mathrm{C}$ for $30 \mathrm{~min}$ and $95^{\circ} \mathrm{C}$ for $5 \mathrm{~min}$. The total volume of the reaction was $25 \mu 1$ and the following components were used: cDNA (2 $\mu 1)$, SYBR Premix Ex Taq $(2 \mathrm{X} ; 10 \mu 1)$, forward primer (10 $\mu \mathrm{M} ; 1 \mu \mathrm{l})$, reverse primer $(10 \mu \mathrm{M} ; 1 \mu \mathrm{l})$, ROX Reference Dye II (1:50 in double-distilled water; $1 \mu 1$ used) and double-distilled water $(10 \mu \mathrm{l})$. The reaction was performed with an initial denaturation step of $30 \mathrm{sec}$ at $95^{\circ} \mathrm{C}$, followed by 40 cycles of denaturation at $95^{\circ} \mathrm{C}$ for $5 \mathrm{sec}$, annealing at $60^{\circ} \mathrm{C}$ for $30 \mathrm{sec}$ and extension at $72^{\circ} \mathrm{C}$ for $30 \mathrm{sec}$. For quantification, all samples were normalized to GAPDH. The mRNA expression levels of each gene were calculated using the $2^{-\Delta \Delta \mathrm{Cq}}$ method (21). The primer sequences were as follows: GAPDH forward, 5'-ATGGCCTTCCGTGTTCCT AC-3' and reverse, 5'-CTTTACAAAGTTGTCGTTGA-3'; ARPC3 forward, 5'-TGCAATTCCAAAAGCCAAGGT-3' and reverse, 5'-AGGCTCTCATCACTTCATCTTCC-3'; HCK forward, 5'-GCAACCGCTGTCATGAGTTAC-3' and reverse, 5'-CTGGTGTGTTGCTGTTGTGG-3'; PIK3CG forward, 5'-GCACTTCCTTCTCGGCTAGAT-3' and reverse, 5'-AATATGAAGCCTGGCTGCCG-3'; PRKCB forward, 5'-GACCAAACACCCAGGCAAAC-3' and reverse, 5'-GAT GGCGGGTGAAAAATCGG-3'; CD209 forward, 5'-TGC TGAGGAGCAGAACTTC-3' and reverse, 5'-GTTGGGCTC TCCTCTGTTCC-3'; CLEC4M forward, 5'-CTCCTGGGG TGTCTTGGC-3' and reverse, 5'-GCGTCTTGCTCGGAT TGTTC-3'; FCGR1A forward, 5'-CGTGAGCACTGCGTA CAAAC-3' and reverse, 5'-TCAGGCTAGGCTTGACTT GT-3'; PTPRC forward, 5'-ACCAGGAATGGATGTCGC TA-3' and reverse, 5'-TGGGGCCTGTAAAAGTGTCC-3'; CYBB forward, 5'-TGTCAAGTGCCCAAAGGTGT-3' and reverse, 5'-CCCAACGATGCGGATATGGA-3'; NCF4 forward, 5'-GAGAGGTGAACTCAGCCTGG-3' and reverse, 5'-TTCAAAGTCACTCTCGGCCC-3'; n337689 forward, 5'-CAGGTAGGCTGGGCCATTAC-3' and reverse, 5'-CCA ATGCCCTTATCCCCCTC-3'; n340778 forward, 5'-GCA GGCATCTGATCCAAGGT-3' and reverse, 5'-AGGGCT AGGCTCAGAATGGA-3'; n341675 forward, 5'-GGCTCC CGGTCTTTCAGAAT-3' and reverse, 5'-TACCCTGTATTG GCCACGTT-3'; n342887 forward, 5'-TCTGTCTGTGCA GTGCTTCTG-3' and reverse, 5'-GTCGTCCTGCAGCAA GTAGC-3'; n345093 forward, 5'-TGGGAGAGGCACACC AACAA-3' and reverse, 5'-CTCCTCGTCCATTTCCGG C-3'; n346352, forward: 5'-GATAAGGGCATTGGGGAG CTG-3' and reverse, 5'-TGTTTTGGGCTTTGCCCCTG-3'; n411602 forward, 5'-TGGGTCTAGCCCACCCAAT-3' and reverse, 5'-CCACTTGGCTCAGATCCACC-3'; NR_002794 forward, 5'-TCTGTCTGTGCAGTGCTTCTG-3' and reverse, 5'-GTCGTCCTGCAGCAAGTAGC-3'; NR_038877 forward, 5'-GGGTGGGATTGGGAGTGTTC-3' and reverse, 5'-TCG GTCCCCTGTTTGAGGTA-3'; NR_039741 forward, 5'-AGG GAGAAGGGTCGGGGC-3' and reverse, 5'-GGGGAGACG TGGGCAGAG-3'. 
Table I. Differential expression of lncRNA in the placental tissues of patients with pre-eclampsia compared with normal pregnancies using microarray analysis.

A, lncRNAs upregulated in patients with pre-eclampsia

\begin{tabular}{llr}
\hline Gene symbol & \multicolumn{2}{c}{ Accession no. } \\
\hline GSTT1 & n336841 & 3.212795 \\
TLT5 & n342887 & 2.190874 \\
SNORA2A & NR_002950 & 2.156267 \\
linc-ADORA2B-1 & TCONS_12_00010577-XLOC_12_005672 \\
TREML5P & NR_002794 & 2.080765 \\
- & ENST00000381105 & 2.053127 \\
- & ENST00000450426 & 1.7945 \\
- & n340625 & 1.746861 \\
LOC100506453 & ENST00000424415 & 1.649479 \\
\hline
\end{tabular}

$\mathrm{B}$, lncRNAs downregulated in patients with pre-eclampsia

\begin{tabular}{lll}
\hline Gene symbol & \multicolumn{2}{c}{ Accession no. } \\
\hline SNORA38 & NR_002971 & -1.51867 \\
TMSB4XP8 & OTTHUMT00000253552 & -1.5274 \\
FCAR & n336109 & -1.54679 \\
IL17RA & n332742 & -1.55508 \\
linc-MED12L & TCONS_00006273-XLOC_002874 \\
- & ENST00000515960 & -1.57362 \\
SNORA11 & NR_002953 & -1.59832 \\
GIMAP4 & n334779 & -1.63376 \\
CD309 & n407224 & -1.63512 \\
VNN2 & n409772 & -1.69438 \\
SNORD14E & ENST00000364009 & -1.71248 \\
- & ENST00000363189 & -1.71758 \\
SNORD113-2 & NR_003230 & -1.80291 \\
SNORA31 & ENST00000534033 & -1.82711 \\
SNORA38B & NR_003706 & -1.82752 \\
IL1RL1 & n333421 & -1.85379 \\
- & ENST00000384564 & -2.09845 \\
\hline
\end{tabular}

lncRNAs, long non-coding RNAs.

Cell proliferation assay. The Cell Counting Kit-8 (CCK-8) assay was used to determine cell proliferation. Lenti-control and lenti-NR_002794 cells were seeded in 96-well plates $\left(2 \times 10^{3}\right.$ cells/well). The cells were incubated in medium with CCK-8 solution (10 $\mu \mathrm{l} / \mathrm{well})$ for $2 \mathrm{~h}$ at $37^{\circ} \mathrm{C}$ following 24,48 and $72 \mathrm{~h}$ of incubation at $37^{\circ} \mathrm{C}$. Absorbance was measured at $450 \mathrm{~nm}$ using a spectrometer (Thermo Fisher Scientific, Inc.).

In vitro cell migration assay. The migratory and invasive abilities of cells were evaluated using the transwell assay in different cell lines. Initially, all transwell chambers were placed in DMEM/Ham's F-12 (1:1; DF-12) medium (Sangon Biotech Co., Ltd.) without serum and were incubated at $37^{\circ} \mathrm{C}$ for $1 \mathrm{~h}$. Blank, lenti-control cells and lenti-NR_002794 cells were harvested and resuspended in DF/12 medium containing
$2 \%$ FBS. Subsequently, $1 \times 10^{4}$ cells were seeded in each upper chamber, while DF/12 medium containing 10\% FBS was added to the lower chambers. Following incubation at $37^{\circ} \mathrm{C}$ for $24 \mathrm{~h}$, the remaining cells on the membrane of the upper chamber surface were gently removed and all chambers were washed with PBS. Cells that had migrated to the lower side of the membrane were treated with $95 \%$ ethanol and stained with crystal violet for $10 \mathrm{~min}$. A fluorescent microscope (magnification, x200; Olympus CX2; Olympus Corporation) was used to examine the number of cells that had migrated, which was calculated from five random fields from each chamber. The fields were used to calculate the average value.

Apoptosis analysis using flow cytometry. To increase the levels of oxidative stress in the cells, $0.01 \% \mathrm{H}_{2} \mathrm{O}_{2}$ was used; this resulted 
in the induction of apoptosis. Following culture with medium containing $0.01 \% \mathrm{H}_{2} \mathrm{O}_{2}$ for $24 \mathrm{~h} 37^{\circ} \mathrm{C}$, the cells were harvested with EDTA-free trypsin. Following washing with PBS, the cells were resuspended in Annexin binding buffer (Thermo Fisher Scientific, Inc.) and double-stained with Annexin V (Nanjing KeyGen Biotech Co., Ltd.) and propidium iodide (PI; Nanjing KeyGen Biotech Co., Ltd.) in the dark for $15 \mathrm{~min}$ at room temperature. Subsequently, the cells were analyzed using a BD FACSCalibur $^{\mathrm{TM}}$ (BD Biosciences). The percentage of apoptotic cells was analyzed using FCAP Array Software (v3.1; BD Biosciences). The analysis resulted in the identification of the four following types of cells: Viable cells (PI negative, Annexin $\mathrm{V}$ negative; lower left quadrant), necrotic cells (PI positive, Annexin V negative; upper left quadrant), early apoptotic cells (PI negative, Annexin V positive; lower right quadrant) and late apoptotic cells (PI positive, Annexin V positive; upper right quadrant). The rate of apoptosis was calculated as the sum of the early apoptotic cells and the late apoptotic cells.

Phagocytosis. The phagocytic capacity of SWAN71 cells following NR_002794 overexpression was determined using a fluorescently labeled pHrodo $^{\mathrm{TM}}$ phagocytic indicator (Thermo Fisher Scientific, Inc.). Cells were incubated with medium containing $1 \mu \mathrm{g} / \mathrm{ml} \mathrm{pHrodo}{ }^{\mathrm{TM}}$, after a $24 \mathrm{~h}$ incubation at $37^{\circ} \mathrm{C}$ the fluorescence intensity was measured using BD FACSCalibur ${ }^{\mathrm{TM}}$ and FCAP Array Software. The fluorescence intensity of the cells (peak area) was also observed using a fluorescent microscope (magnification, x200).

Statistical analysis. All data were analysed with SPSS 23.0 (IBM Corp.) and GraphPad Prism 5 (GraphPad Software, Inc., La Jolla, CA, USA). The data are presented as the mean \pm SD. All experiments were repeated three times. Comparisons between two groups were analyzed using independent sample t-tests. One-way ANOVA followed by Dunnett's test was used for the comparison of multiple samples. $\mathrm{P}<0.05$ was considered to indicate a statistically significant difference.

\section{Results}

Differentially expressed lncRNAs and mRNAs in human trophoblast cells. Based on microarray and computational analysis, 16 lncRNAs were identified as being differentially expressed between human trophoblast cells from patients with pre-eclampsia and those without pre-eclampsia, including 9 that were upregulated and 17 that were downregulated (Table I). Furthermore, the profiles of differentially expressed mRNAs are shown in Table II. The data indicated that 121 mRNAs were downregulated and $87 \mathrm{mRNAs}$ were upregulated in the placental tissue of patients with pre-eclampsia compared with the expression of these molecules in the placental tissue from normal pregnancies; Table II presents the top 10 upregulated and 10 downregulated mRNAs. GO and KEGG analyses were performed to reveal the relationship between the differentially expressed mRNAs and their biological functions (Fig. 1A-D). These results indicated that these genes identified were involved in invasion, metastasis and phagocytosis. In addition, according to the interactions between lncRNAs and mRNAs, NR_002794 was identified as an upregulated lncRNA with
Table II. Differential expression of mRNA in in the placental tissues of patients with pre-eclampsia compared with normal pregnancies using microarray analysis.

A, Genes upregulated in patients with pre-eclampsia

\begin{tabular}{lll}
\hline Gene symbol & Accession no. & Fold-change \\
\hline TREML2 & NM_024807 & 2.548855 \\
ALAS2 & NM_000032 & 2.513751 \\
IFIT1B & NM_001010987 & 2.513519 \\
GTSF1 & NM_144594 & 2.506887 \\
KIAA1199 & NM_018689 & 1.724283 \\
LY6G6C & NM_025261 & 1.611992 \\
SLC4A1 & NM_000342 & 1.605567 \\
GSTT1 & NM_000853 & 1.438739 \\
DERL3 & NM_001002862 & 1.41813 \\
HOOK1 & NM_015888 & 1.36205 \\
\hline
\end{tabular}

$\mathrm{B}$, Genes downregulated in patients with pre-eclampsia

\begin{tabular}{llr}
\hline Gene symbol & Accession no. & Fold-change \\
\hline CYTL1 & NM_018659 & -1.95817 \\
IL1RL1 & NM_003856 & -1.87074 \\
MRC1 & NM_002438 & -1.79006 \\
MRC1 & NM_002438 & -1.76872 \\
CD209 & NM_001144893 & -1.71566 \\
VSIG4 & NM_001100431 & -1.58222 \\
FKBP5 & NM_004117 & -1.55752 \\
CLEC4M & NM_001144904 & -1.53693 \\
DPYD & NM_000110 & -1.49611 \\
CYBB & NM_000397 & -1.49244 \\
\hline
\end{tabular}

a role in the induction of apoptosis and migration (Fig. 1E). Thus, the function of NR_002794 in pre-eclampsia was investigated in the present study.

Validation using $R T$ - $q P C R$. The lncRNA and mRNA expression levels were compared in placental tissues from 3 patients with pre-eclampsia and in tissues from 3 normal pregnancies using RT-qPCR. According to the GO and KEGG analyses, the expression levels of 10 mRNAs and 10 lncRNAs were detected in placental samples. As shown in Fig. 2A, the expression levels of several mRNAs (actin related protein $2 / 3$ complex subunit 3, phosphatidylinositol-4,5-bisphosphate 3-kinase catalytic subunit $\gamma$, C-type lectin domain family 4 member $M$, Fc fragment of IgG receptor 1a, cytochrome b-245 $\beta$ chain and neutrophil cytosolic factor 4) were downregulated in patients with pre-eclampsia, whereas the expression levels of several lncRNAs (n340778, n342887, n345093, n346352, NR_002794, NR_038877 and NR_039741) were increased in patients with pre-eclampsia (Fig. 2B).

Overexpression of NR_002794 in human SWAN71 trophoblast cells. To further examine the function of NR_002794 
A

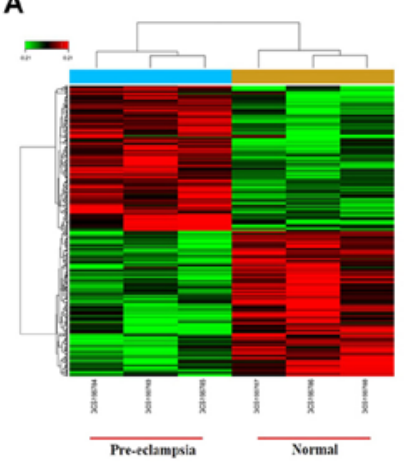

D

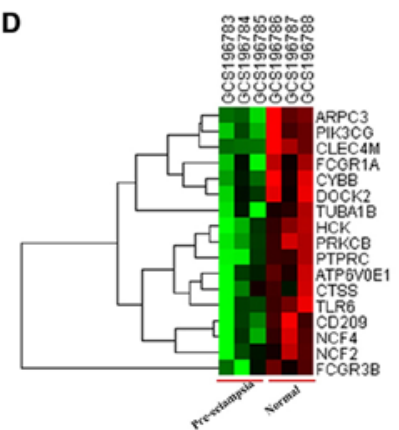

B

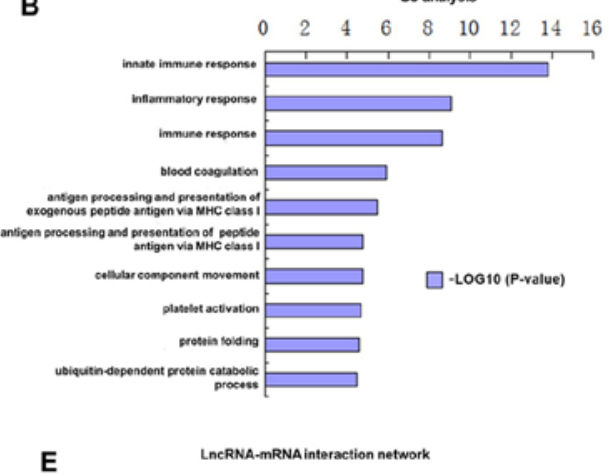

E

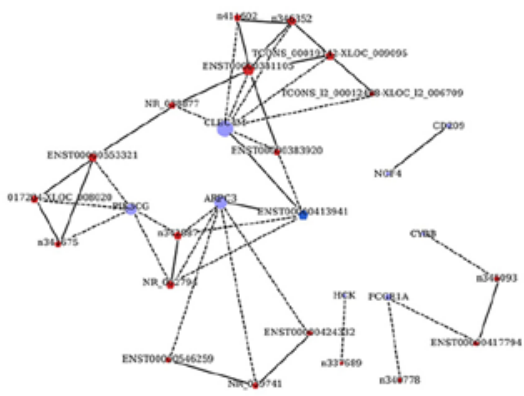

C

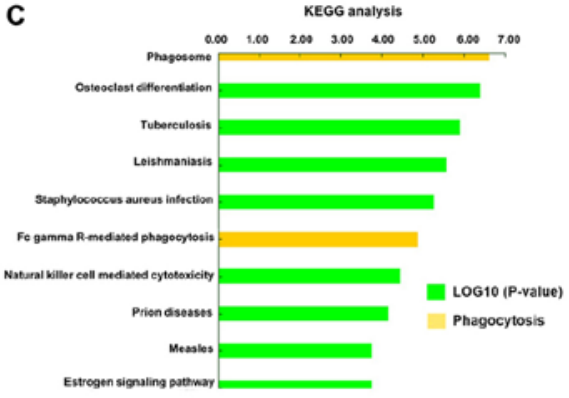

Figure 1. Analysis of the expression profiles of differentially expressed mRNAs and lncRNAs in the placental tissues of patients with pre-eclampsia and normal pregnancies using Affymetrix microarray technology. (A) Heat map constructed using differentially expressed genes. Red indicates upregulation and green indicates downregulation of the genes compared with the average level of expression. (B) GO pathway analysis of the differentially expressed genes. The top $10 \mathrm{GO}$ terms are listed. (C) KEGG pathway analysis of differentially expressed genes. The top 10 KEGG pathways identified are listed. (D) Cluster analysis of differentially expressed genes. (E) lncRNA-mRNA interaction network. The circles represent mRNAs and the pentagons represent lncRNAs. Red indicates upregulation while blue indicates downregulation. Solid lines indicate a positive correlation and dashed lines indicate a negative correlation. IncRNA, long noncoding RNA; GO, Gene Ontology, KEGG, Kyoto Encyclopedia of Genes and Genomes.

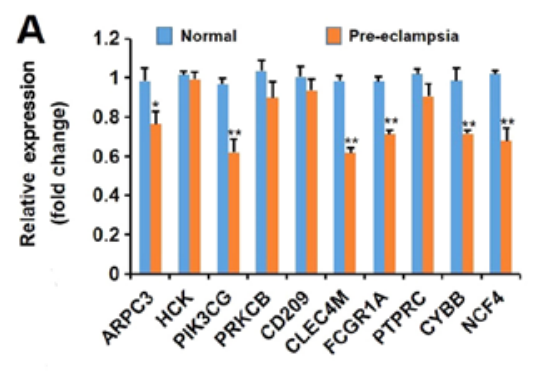

D

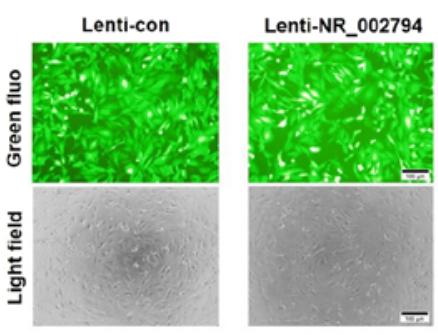

B

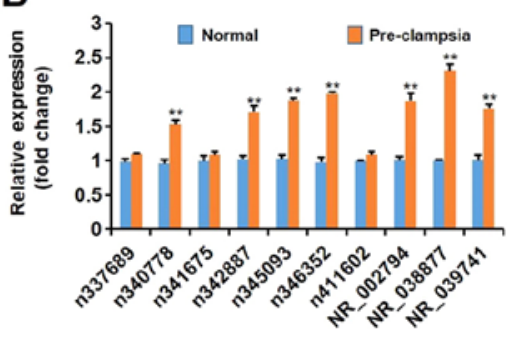

E

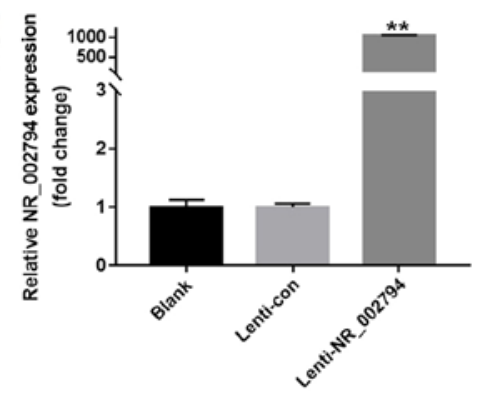

C
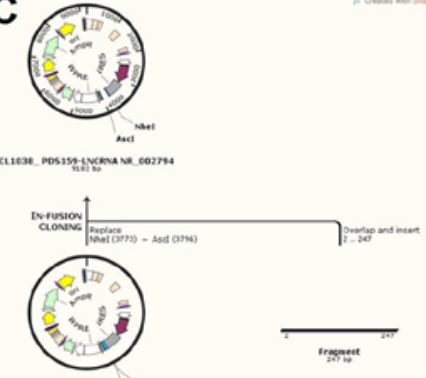

posisonas.

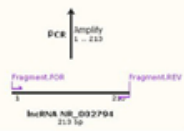

Figure 2. RT-qPCR verification of differentially expressed genes identified using microarray analysis and the overexpression of NR_002794 in SWAN71 cells. (A) The expression levels of 10 mRNAs (ARPC3, HCK, PIK3CG, PRKCB, CD209, CLEC4M, FCGR1A, PTPRC, CYBB and NCF4) were determined by RT-qPCR in tissues derived from pre-eclamptic pregnancies and normal pregnancies. (B) The expression levels of 10 lncRNAs (n337689, n340778, n341675, n342887, n345093, n346352, n411602, NR_002794, NR_038877 and NR_039741) were determined by RT-qPCR in tissues derived from pre-eclamptic pregnancies and normal pregnancies. ${ }^{*} \mathrm{P}<0.05 ;{ }^{* *} \mathrm{P}<0.01$ vs. respective normal group. (C) Schematic representation of the construction of the overexpression plasmid containing the full-length lncRNA NR_002794. (D) Lentiviral infection of SWAN71 cells. Cells that were infected with lenti-NR_002794 and lenti-con exhibited a consistent infection efficiency. Magnification, x200. (E) SWAN71 cells were infected with lenti-NR_002794 or lenti-con and the infection efficiency was assessed using RT-qPCR. The data are presented as the mean $\pm \mathrm{SD}$. ${ }^{* *} \mathrm{P}<0.01$ vs. lenti-con. RT-qPCR, reverse transcription-quantitative PCR; ARPC3, actin related protein 2/3 complex subunit 3; HCK, HCK proto-oncogene Src family tyrosine kinase; PIK3CG, phosphatidylinositol-4,5-bisphosphate 3-kinase catalytic subunit $\gamma$; PRKCB, protein kinase C $\beta$; CD209, CD209 molecule; CLEC4M, C-type lectin domain family 4 member M; FCGR1A, Fc fragment of IgG receptor 1a; PTPRC, protein tyrosine phosphatase receptor type C; CYBB, cytochrome b-245 $\beta$ chain; NCF4, neutrophil cytosolic factor 4; lenti-NR_002794, lentivirus expressing NR_002794; lenti-con, control lentivirus; fluo, fluorescence. 
A

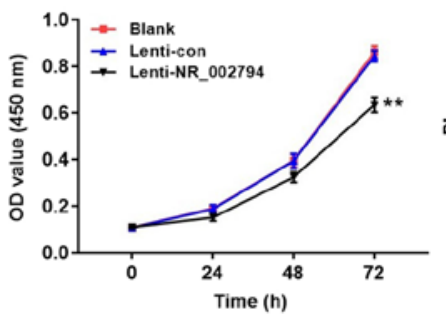

B

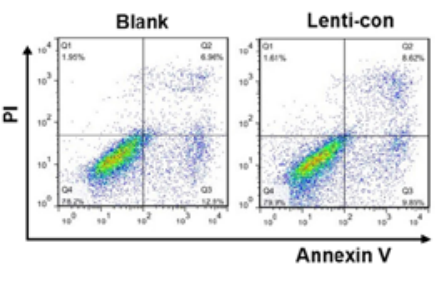

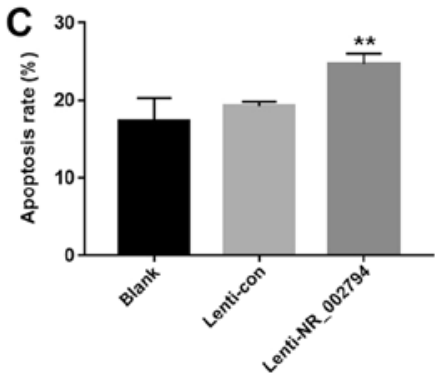

Figure 3. Effect of NR_002794 expression on the proliferation and apoptosis of trophoblast cells. (A) SWAN71 cells were infected with lenti-control or lenti-NR_002794 for 24, 48 or $72 \mathrm{~h}$. Cell proliferation was determined using the Cell Counting Kit-8 assay. (B) The rate of apoptosis in SWAN71 cells infected with lenti-control or lenti-NR_002794 for $72 \mathrm{~h}$ was determined using flow cytometry. (C) Statistical analysis of the rate of apoptosis. Data is presented as the mean \pm SD. ${ }^{* *} \mathrm{P}<0.01$ vs. lenti-con. lenti-NR_002794, lentivirus expressing NR_002794; lenti-con, control lentivirus; OD, optical density; PI, propidium iodide.

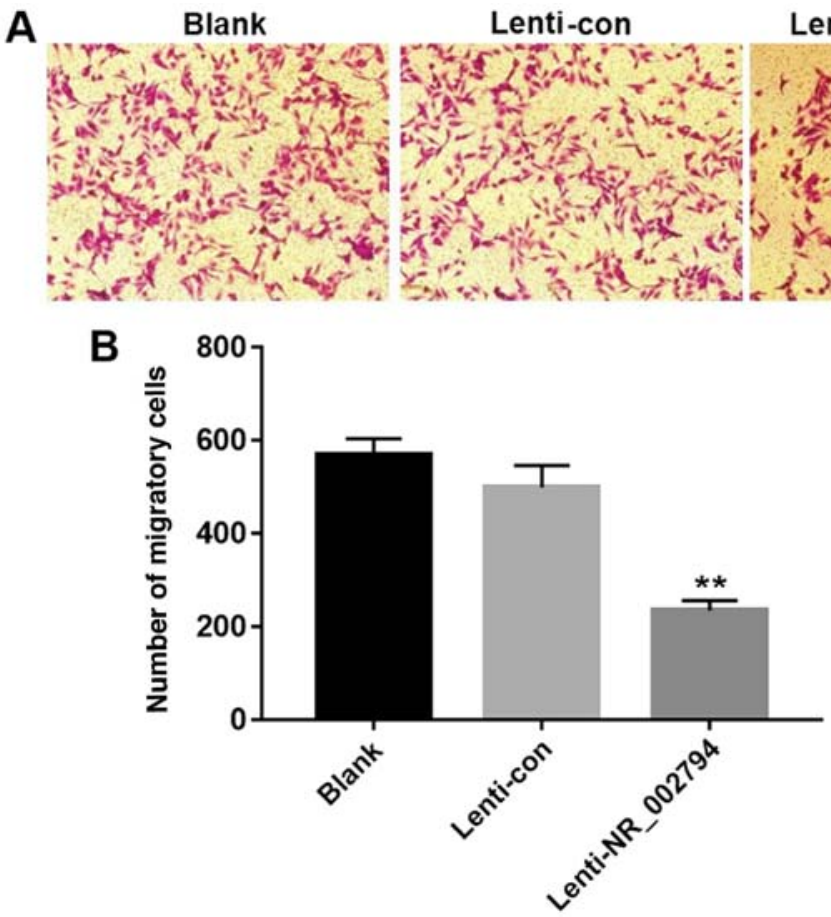

Figure 4. Effect of NR_002794 expression on the migration of trophoblast cells. (A) SWAN71 cells were infected with lenti-con or lenti-NR_002794 for $24 \mathrm{~h}$. The migration of trophoblast cells was determined using transwell assays. Magnification, x200. (B) Statistical analysis of the migration of cells. Data is presented as the mean $\pm \mathrm{SD}$. ${ }^{* *} \mathrm{P}<0.01$ vs. lenti-con. lenti-NR_002794, lentivirus expressing NR_002794; lenti-con, control lentivirus.

in the behavior of trophoblast cells, an increase in the expression of NR_002794 was achieved using an expression vector in SWAN71 cells (Fig. 2C). Cells that were infected with lenti-NR_002794 or lenti-control exhibited a consistent infection efficiency (Fig. 2D). Following infection with lenti-NR_002794 or lenti-control, the cells were harvested to determine the level of overexpression using RT-qPCR. Significant overexpression of NR_002794 was detected in lenti-NR_002794 cells compared with the lenti-control cells (Fig. 2E).

Overexpression of NR_002794 inhibits the proliferation of trophoblast cells. The significant increase in the expression of NR_002794 in the placental tissues of patients with pre-eclampsia prompted an investigation into the possible biological functions of NR_002794 in pre-eclampsia. To investigate the effects of NR_002794 on the proliferation of trophoblast cells, the CCK-8 assay was used following the infection of SWAN71 cells with lenti-control or lenti-NR_002794 vectors. Infection with lenti-NR_002794 significantly decreased cell proliferation compared with the blank and lenti-control groups (Fig. 3A; $\mathrm{P}<0.01$ ). These results suggested that the increased expression of NR_002794 inhibited the proliferation of SWAN71 cells.

Overexpression of NR_002794 promotes the apoptosis of trophoblast cells. Flow cytometry was performed to detect the effects of NR_002794 expression on the level of apoptosis in trophoblast cells. The infection of SWAN71 cells with lenti-NR_002794 caused a significant increase in the induction of apoptosis compared with the lenti-control and blank groups (Fig. 3B and $\mathrm{C} ; \mathrm{P}<0.01$ ). These results indicated that NR_002794 promoted the induction of apoptosis. 

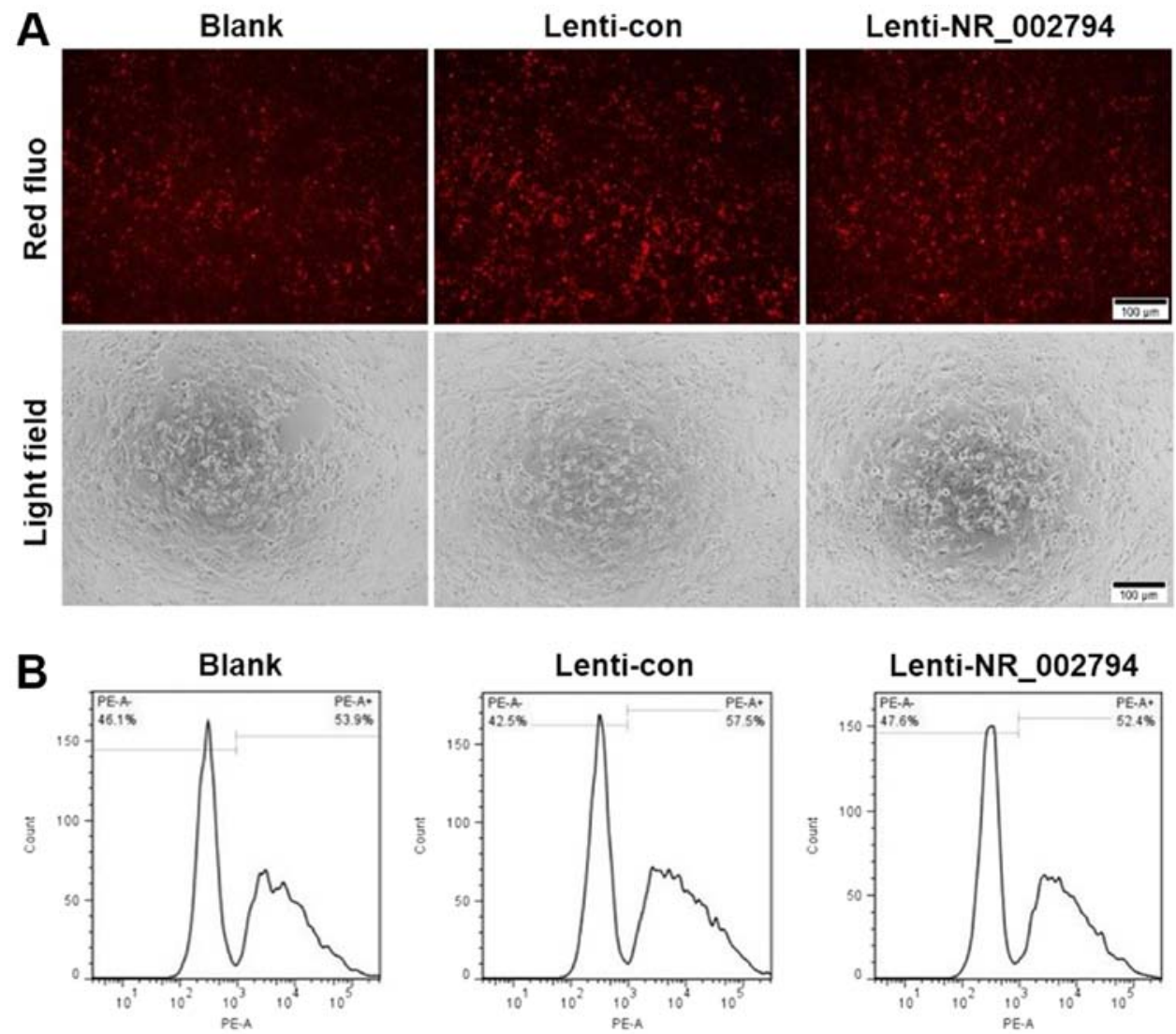

Figure 5. Effect of NR_002794 expression on the phagocytic activity of trophoblast cells. SWAN71 cells were infected with lenti-con or lenti-NR_002794 for $72 \mathrm{~h}$. (A) The phagocytic activity of SWAN71 cells in each group was determined using fluorescence microscopy. Magnification, x200. (B) The effect of NR_002794 on the phagocytic activity of SWAN71 cells was determined using flow cytometry. lenti-NR_002794, lentivirus expressing NR_002794; lenti-con, control lentivirus; fluo, fluorescence.

Overexpression of $N R \_002794$ inhibits the migration of trophoblast cells. The transwell assay was used to investigate the effects of NR_002794 expression on the migration of SWAN71 cells. The results indicated that expression of NR_002794 significantly decreased the number of migrating cells compared with the blank and lenti-control groups (Fig. 4), indicating that NR_002794 inhibited the migration of trophoblast cells. Cells expressing lenti-NR_002794 exhibited no significant difference in proliferation compared with the blank and lenti-control groups at $24 \mathrm{~h}$. Thus, the inhibitory effect of lenti-NR_002794 on migration was not a result of cell death.

Overexpression of NR_002794 does not affect the phagocytic activity of trophoblast cells. SWAN71 cells that overexpressed NR_002794 exhibited no significant difference in their phagocytic activity compared with the blank and lenti-control groups (Fig. 5). This was demonstrated by fluorescence and flow cytometry analysis. These data suggested that NR_002794 did not affect the phagocytic activity of trophoblast cells.

\section{Discussion}

Pre-eclampsia is characterized by hypertension and proteinuria. It is a common and severe complication encountered during pregnancy $(22,23)$. Patients with pre-eclampsia often suffer from increased uric acid levels and serum transaminase concentrations (24). Currently, the only effective treatment for pre-eclampsia is delivery or interruption of pregnancy (25). The pathogenesis of pre-eclampsia remains elusive. The failure of cells to infiltrate into the trophoblast and the abnormal development of the placenta are considered to be the main factors in the development of this disease (26). EVTs differentiate and invade the maternal endometrium for intravascular transformation, forming normal placental vessels. However, trophoblastic cellular dysfunction or the failure of invasion can lead to maternal spiral artery-remodeling failure $(11,12)$. The differentiation of trophoblast cells is regulated by various factors and the regulatory role of lncRNAs has received attention (8).

It has been shown that $>90 \%$ of the human genome does not encode protein products: The transcripts of these genes are called non-protein-encoding RNAs (ncRNAs) (27). ncRNAs are broadly divided into two major classes: microRNAs and lncRNAs (28). With the advancement of transcriptomics, the role of lncRNAs in various cellular activities and ontogeny is being recognized (29). lncRNAs are capable of regulating the development and metabolism of several cellular activities at the epigenetic, transcriptional and post-transcriptional levels (30). A number of studies have shown that the abnormal expression of lncRNAs in placental tissues during pregnancy is closely related to the onset of pre-eclampsia $(31,32)$. He et al $(16)$ reported that 
738 lncRNAs were differentially expressed in the placental tissues of patients with pre-eclampsia, including 479 that were upregulated and 259 that were downregulated. These differentially expressed lncRNAs were identified following the microarray analysis of tissues derived from 6 patients with pre-eclampsia and 5 normal pregnancies (16). Zou et al (11) examined the expression levels of specific lncRNAs in the placental tissues of 25 patients with pre-eclampsia and found a 2.8 -fold increase in the expression level of the lncRNA SPRY4-IT1 compared with placental tissues from normal pregnancies. Moreover, the increased expression of SPRY4-IT1 was found to reduce the rate of invasion, metastasis and proliferation of trophoblast cells and increase the rate of apoptosis. Zhang et al (12) demonstrated that the expression level of the lncRNA MEG3 in the placental tissues from patients with pre-eclampsia were significantly lower than those from normal pregnancies. The low expression of MEG3 promoted the induction of trophoblast cell apoptosis and inhibited the invasive and metastatic abilities of the cells. Chen et al (17) further showed that MALAT-1, which is considered to be a significant tumor marker, played an important role in trophoblast cell invasion and metastasis.

In the present study, tissues from 3 cases of pre-eclampsia and 3 cases of normal pregnancy were investigated. A total of 26 lncRNAs with differential expression levels were identified, this included 9 upregulated lncRNAs and 17 downregulated lncRNAs. In addition, 208 differentially expressed mRNAs were identified that were classified as upregulated (87) or downregulated (121). GO and KEGG pathway analyses were performed and indicated that these genes were involved in invasion, metastasis and phagocytosis. In the present study, NR_002794, a lncRNA that was found to be highly expressed in pre-eclampsia, was selected as a target to investigate the effects of lncRNAs on the function of the trophoblast cell line SWAN71. It was observed that the upregulation of NR_002794 suppressed the proliferation and migration of SWAN71 cells, and increased the induction of apoptosis. As such, it is hypothesized that NR_002794 may play an important role in the proliferation, migration and apoptosis of trophoblast cells in pre-eclampsia. NR_002794 may play an important role in trophoblast cell activity, in the remodeling of the uterine spiral artery and in the normal development of the placenta. Abnormal expression of NR_002794 may inhibit the invasion of trophoblast cells into the maternal endometrium, resulting in insufficient remodeling of the spiral arteries, incomplete development of the placenta and insufficient perfusion. These processes eventually lead to the development of pre-eclampsia. However, the molecular mechanism underlying the role of NR_002794 with regards to trophoblast invasion and apoptosis remains unclear and requires further investigation.

In conclusion, the present study demonstrated that lncRNA NR_002794 was upregulated in the placental tissues of patients with pre-eclampsia. The results further showed that the expression levels of NR_002794 may alter the biological functions of trophoblast cells in vitro.

\section{Acknowledgements}

Not applicable.

\section{Funding}

The present study was supported by the Project of Guangxi Natural Science Foundation (grant nos. 2017GXNSFAA198084 and 2014GXNSFBA118176).

\section{Availability of data and materials}

The datasets used and/or analyzed during the current study are available from the corresponding author on reasonable request.

\section{Authors' contributions}

YiM and XL performed experiments, analyzed data and were major contributors in the development of the manuscript. HW and CZ collected tissues, interpreted patient data and performed experiments. YaM interpreted patient data and reviewed the final version of the manuscript.

\section{Ethics approval and consent to participate}

Ethical approval for the present study was received from the People's Hospital of Guangxi Zhuang Autonomous Region. Written consent was obtained from all donors.

\section{Patient consent for publication}

All patients provided written informed consent for the publication of all associated information.

\section{Competing interests}

The authors declare that they have no competing interests.

\section{References}

1. Mol BWJ, Roberts CT, Thangaratinam S, Magee LA, de Groot CJM and Hofmeyr GJ: Pre-eclampsia. Lancet 387: 999-1011, 2016.

2. Ahn S, Jeong E, Min JW, Kim E, Choi SS, Kim CJ and Lee DC: Identification of genes dysregulated by elevation of microRNA-210 levels in human trophoblasts cell line, Swan 71. Am J Reprod Immunol 78: e12722, 2017.

3. Souza JP, Gulmezoglu AM, Vogel J, Carroli G, Lumbiganon P, Qureshi Z, Costa MJ, Fawole B, Mugerwa Y, Nafiou I, et al: Moving beyond essential interventions for reduction of maternal mortality (the WHO Multicountry Survey on Maternal and Newborn Health): A cross-sectional study. Lancet 381: 1747-1755, 2013.

4. Hansen AR, Barnes CM, Folkman J and McElrath TF: Maternal preeclampsia predicts the development of bronchopulmonary dysplasia. J Pediatr 156: 532-536, 2010.

5. Strand KM, Heimstad R, Iversen AC, Austgulen R, Lydersen S, Andersen GL, Irgens LM and Vik T: Mediators of the association between pre-eclampsia and cerebral palsy: Population based cohort study. BMJ 347: f4089, 2013.

6. Irgens HU, Reisaeter L, Irgens LM and Lie RT: Long term mortality of mothers and fathers after pre-eclampsia: Population based cohort study. BMJ 323: 1213-1217, 2001.

7. Huppertz B: Placental origins of preeclampsia: Challenging the current hypothesis. Hypertension 51: 970-975, 2008.

8. Du MR, Wang SC and Li DJ: The integrative roles of chemokines at the maternal-fetal interface in early pregnancy. Cell Mol Immunol 11: 438-448, 2014.

9. Liu X, Chen H, Kong W, Zhang Y, Cao L, Gao L and Zhou R: Down-regulated long non-coding RNA-ATB in preeclampsia and its effect on suppressing migration, proliferation, and tube formation of trophoblast cells. Placenta 49: 80-87, 2017. 
10. Chakraborty C, Gleeson LM, McKinnon T and Lala PK: Regulation of human trophoblast migration and invasiveness. Can J Physiol Pharmacol 80: 116-124, 2002.

11. Zou Y, Jiang Z, Yu X, Sun M, Zhang Y, Zuo Q, Zhou J, Yang N, Han P, Ge Z, et al: Upregulation of long noncoding RNA SPRY4-IT1 modulates proliferation, migration, apoptosis, and network formation in trophoblast cells HTR-8SV/neo. PLoS One 8: e79598, 2013.

12. Zhang Y, Zou Y, Wang W, Zuo Q, Jiang Z, Sun M, De W and Sun L: Down-regulated long non-coding RNA MEG3 and its effect on promoting apoptosis and suppressing migration of trophoblast cells. J Cell Biochem 116: 542-550, 2015.

13. Akhade VS, Pal D and Kanduri C: Long noncoding RNA: Genome organization and mechanism of action. Adv Exp Med Biol 1008: 47-74, 2017.

14. Guo X and Hua Y: CCAT1: An oncogenic long noncoding RNA in human cancers. J Cancer Res Clin Oncol 143: 555-562, 2017.

15. Johnson R: Long non-coding RNAs in Huntington's disease neurodegeneration. Neurobiol Dis 46: 245-254, 2012.

16. He X, He Y, Xi B, Zheng J, Zeng X, Cai Q, Ouyang Y, Wang C Zhou X, Huang $\mathrm{H}$, et al: LncRNAs expression in preeclampsia placenta reveals the potential role of LncRNAs contributing to preeclampsia pathogenesis. PLoS One 8: e81437, 2013.

17. Chen H, Meng T, Liu X, Sun M, Tong C, Liu J, Wang H and Du J: Long non-coding RNA MALAT-1 is downregulated in preeclampsia and regulates proliferation, apoptosis, migration and invasion of JEG-3 trophoblast cells. Int J Clin Exp Pathol 8: 12718-12727, 2015.

18. Zou YF and Sun LZ: Long noncoding RNA HOTAIR modulates the function of trophoblast cells in pre-eclampsia. Sichuan Da Xue Xue Bao Yi Xue Ban 46: 113-117, 122, 2015 (In Chinese)

19. Gao C, Zhao D, Zhao Q, Dong D, Mu L, Zhao X, Guo M, Xu A, Fang L, Liu Q and Che J: Microarray profiling and co-expression network analysis of IncRNAs and mRNAs in ovarian cancer. Cell Death Discov 5: 93, 2019.

20. Mlecnik B, Galon J and Bindea G: Comprehensive functional analysis of large lists of genes and proteins. J Proteomics 171: 2-10, 2018.

21. Livak KJ and Schmittgen TD: Analysis of relative gene expression data using real-time quantitative PCR and the 2(-Delta Delta C(T)) method. Methods 25: 402-408, 2001.

22. Ray JG, Vermeulen MJ, Schull MJ and Redelmeier DA: Cardiovascular health after maternal placental syndromes (CHAMPS): Population-based retrospective cohort study. Lancet 366: 1797-1803, 2005 .
23. ACOG Committee on Practice Bulletins-Obstetrics: ACOG practice bulletin. Diagnosis and management of preeclampsia and eclampsia. Number 33, January 2002. Obstet Gynecol 99: 159-167, 2002.

24. Álvarez-Cabrera MC, Barrientos-Galeana E, Barrera-García A, Osorio-Caballero M, Acevedo JF, Flores-Herrera O, Díaz NF, Molina-Hernández A, García-López G and Flores-Herrera H: Secretion of heat shock $-60,-70 \mathrm{kD}$ protein, IL-1 $\beta$ and $\mathrm{TNF} \alpha$ levels in serum of a term normal pregnancy and patients with pre-eclampsia development. J Cell Mol Med 22: 5748-5752, 2018.

25. Schlichting LE, Insaf TZ,Zaidi AN, Lui GK and Van Zutphen AR: Maternal comorbidities and complications of delivery in pregnant women with congenital heart disease. J Am Coll Cardiol 73: 2181-2191, 2019.

26. Heazell AE, Buttle HR, Baker PN and Crocker IP: Altered expression of regulators of caspase activity within trophoblast of normal pregnancies and pregnancies complicated by preeclampsia. Reprod Sci 15: 1034-1043, 2008

27. Gloss BS and Dinger ME: The specificity of long noncoding RNA expression. Biochim Biophys Acta 1859: 16-22, 2016.

28. Shi Q and Yang X: Circulating MicroRNA and long noncoding RNA as biomarkers of cardiovascular diseases. J Cell Physiol 231: 751-755, 2016.

29. Zhang Z, Tang J, Di R, Liu Q, Wang X, Gan S, Zhang X, Zhang J, $\mathrm{Hu}$ W and Chu M: Comparative transcriptomics reveal key sheep (Ovis aries) hypothalamus LncRNAs that affect reproduction. Animals (Basel) 9: pii: E152, 2019.

30. Sun W, Yang Y, Xu C and Guo J: Regulatory mechanisms of long noncoding RNAs on gene expression in cancers. Cancer Genet 216-217: 105-110, 2017.

31. Pengjie Z, Xionghui C, Yueming Z, Ting X, Na L, Jianying T and Zhice X: LncRNA uc003fir promotes CCL5 expression and negatively affects proliferation and migration of trophoblast cells in preeclampsia. Pregnancy Hypertens 14: 90-96, 2018.

32. Yang Y, Xi L, Ma Y, Zhu X, Chen R, Luan L, Yan J and An R The lncRNA small nucleolar RNA host gene 5 regulates trophoblast cell proliferation, invasion, and migration via modulating miR-26a-5p/N-cadherin axis. J Cell Biochem 120: 3173-3184, 2019.

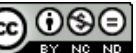

This work is licensed under a Creative Commons Attribution-NonCommercial-NoDerivatives 4.0 International (CC BY-NC-ND 4.0) License. 